Int Arch Allergy Immunol 2021;182:76-82

DOI: $10.1159 / 000510953$
Received: July 30, 2020

Accepted: August 16, 2020

Published online: September 22, 2020

\title{
Does Asthma Increase the Mortality of Patients with COVID-19?: A Systematic Review and Meta-Analysis
}

\author{
Yuanyuan Wang ${ }^{a}$ Jingjing Chen ${ }^{a}$ Wei Chen ${ }^{b}$ Ling Liu ${ }^{b}$ Mei Dong ${ }^{b}$ Juan Ji ${ }^{b}$ \\ Die Huc Nianzhi Zhang ${ }^{\mathrm{b}}$ \\ ${ }^{a}$ Graduate School, Anhui University of Chinese Medicine, Hefei, China; ${ }^{b}$ Department of Respiratory Medicine, \\ The First Affiliated Hospital of Anhui University of Chinese Medicine, Hefei, China; ' Department of Scientific \\ Research, The First Affiliated Hospital of Anhui University of Chinese Medicine, Hefei, China
}

\section{Keywords}

COVID-19 · Coronavirus · Asthma · Respiratory disease ·

Death

\begin{abstract}
The purpose of this systematic review and meta-analysis was to explore the literature and collate data comparing the mortality of coronavirus disease 2019 (COVID-19) patients with and without asthma. The databases PubMed, Scopus, Embase, Google Scholar, and medRxiv.org were searched for studies comparing the clinical outcomes of asthmatic patients with those of nonasthmatic patients diagnosed with COVID-19. Mortality data were summarized using the Mantel-Haenszel OR with $95 \% \mathrm{Cl}$ in a random-effects model. Five retrospective studies met the inclusion criteria. A meta-analysis of data from 744 asthmatic patients and 8,151 nonasthmatic patients indicated that the presence of asthma had no significant effect on mortality (OR $=0.96 ; 95 \% \mathrm{Cl} 0.70-1.30$; $R^{2}=0 \% ; p=0.79$ ). Results were stable in a sensitivity analysis. A descriptive analysis of other clinical outcomes indicated no difference in the duration of hospitalization and the risk of intensive care unit (ICU) transfer between asthmatic and nonasthmatic patients. To conclude, preliminary data indi-
\end{abstract}

cates that asthma as a comorbidity may not increase the mortality of COVID-19. Data on the influence of asthma on the risk of hospitalization, the duration of hospitalization, the requirement of ICU admission, and disease severity is still too limited to draw any strong conclusions. Further studies with a larger sample size are required to establish strong evidence.

(c) 2020 S. Karger AG, Basel

\section{Introduction}

The severe acute respiratory syndrome coronavirus 2 (SARS-CoV-2) causing the coronavirus disease 2019 (COVID-19) was first detected in December 2019. Since then, COVID-19 has rapidly infected patients in several countries worldwide, leading to an immense strain on healthcare systems [1]. Patients with this disease commonly present with symptoms like fever, fatigue, dry cough, sore throat, and difficulty breathing [2]. A number of patients may also develop lymphopenia and pneumonia with characteristic findings on chest computed tomography (CT) [3]. Several clinical studies have identified comorbidities like hypertension, diabetes mellitus, karger@karger.com

www.karger.com/iaa

Karger
(C) 2020 S. Karger AG, Basel

(n)
Nianzhi Zhang

Department of Respiratory Medicine

The First Affiliated Hospital of Anhui University of Chinese Medicine

No. 117 Meishan Rd., Hefei 230031 (China)

dczhangnz@126.com 


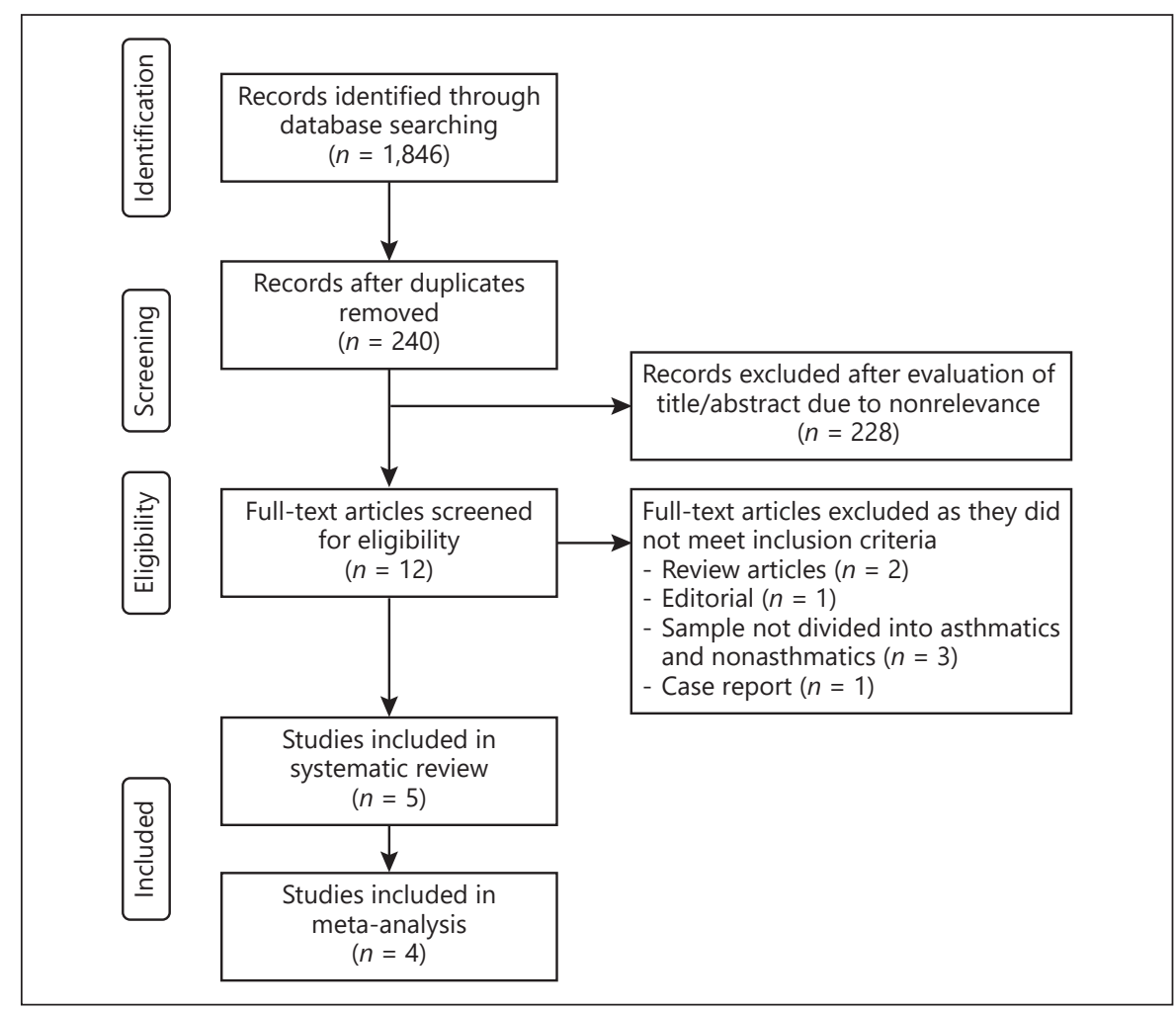

chronic obstructive pulmonary disease, cardiovascular disease, and obesity as risk factors for worsening of outcomes with COVID-19 [4, 5]. Nevertheless, with increasing cases and reporting of clinical data, the knowledge of the disease is continuously evolving.

Asthma is a common pulmonary disease, affecting around $8-9 \%$ of the population of the USA [6]. It is well known that viral infections can lead to exacerbation of asthma [7]; however, it is still unclear whether asthma leads to an increased susceptibility to COVID-19 or deterioration of clinical outcomes [8]. In one of the earliest studies in 140 Chinese patients, Zhang et al. [9] reported that allergic diseases and asthma are not risk factors for the development of COVID-19 infection. However, the US Centers for Disease Control and Prevention (CDC) has advised that moderately to severely asthmatic patients may have a higher risk of COVID-19, although there is no current evidence to support that statement [10].

Since SARS-CoV-2 predominantly affects the lungs, there is a need to understand whether chronic respiratory diseases like asthma increase the mortality of COVID-19 patients. Therefore, the purpose of this systematic review and meta-analysis is to explore the literature and collate data comparing the mortality of COVID-19 patients with and without asthma.

\section{Materials and Methods}

\section{Inclusion Criteria}

The protocol was not registered. Both peer-reviewed studies and non-peer-reviewed studies were to be included. For inclusion in this review, studies were to compare the clinical outcomes of asthmatic patients with those of nonasthmatic patients diagnosed with COVID-19 by RT-PCR. No restriction was placed on the study type. We excluded: (1) studies not dividing the cohort into asthmatics and nonasthmatics; (2) studies not reporting clinical outcomes; (3) studies with fewer than 10 patients in the asthmatic group; (4) case series, case reports, and review articles; and (5) non-English language studies. In the case of studies with overlapping data, the study reporting the largest data set was included.

\section{Search Strategy}

We performed an electronic search in the databases PubMed, Scopus, Embase, and Google Scholar for peer-reviewed articles. The database of MedRxiv.org was searched for any preprints meeting the inclusion criteria. The last search was performed on June 15, 2020. Two independent reviewers carried out the database search using the following keywords in various combinations: "COVID-19," "Coronavirus," "COVID," "SARS-CoV-2," and "asthma." After selection of the studies, a manual check of the bibliography of all of the included studies was performed to check for any missed studies. Search records were first screened by their titles and abstracts by 2 different reviewers. After the abstract screening, the full texts of relevant articles were extracted and assessed in detail based on the inclusion criteria. Any disagreements during this study were resolved by discussion and mutual consensus.

Int Arch Allergy Immunol 2021;182:76-82 DOI: $10.1159 / 000510953$ 


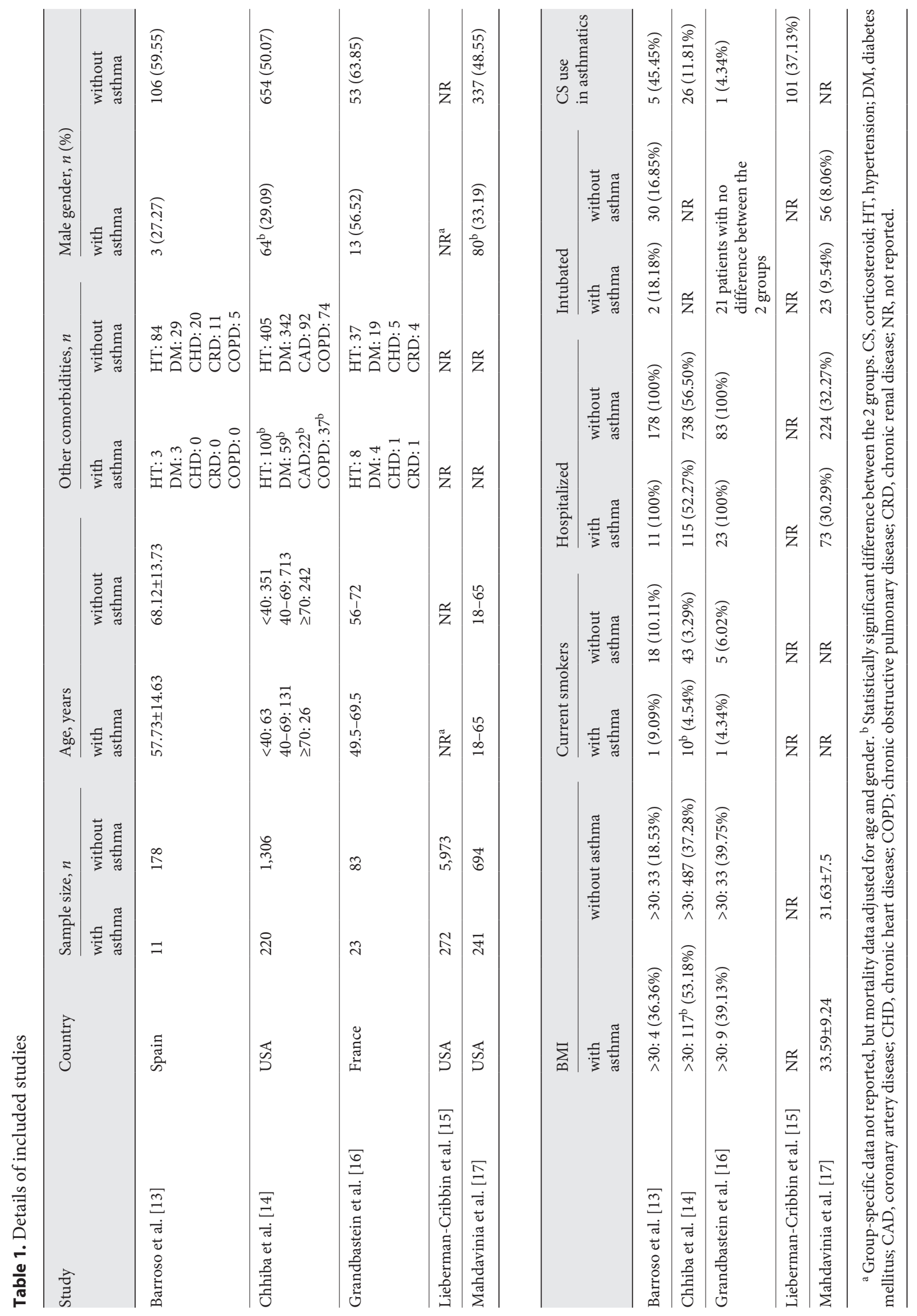




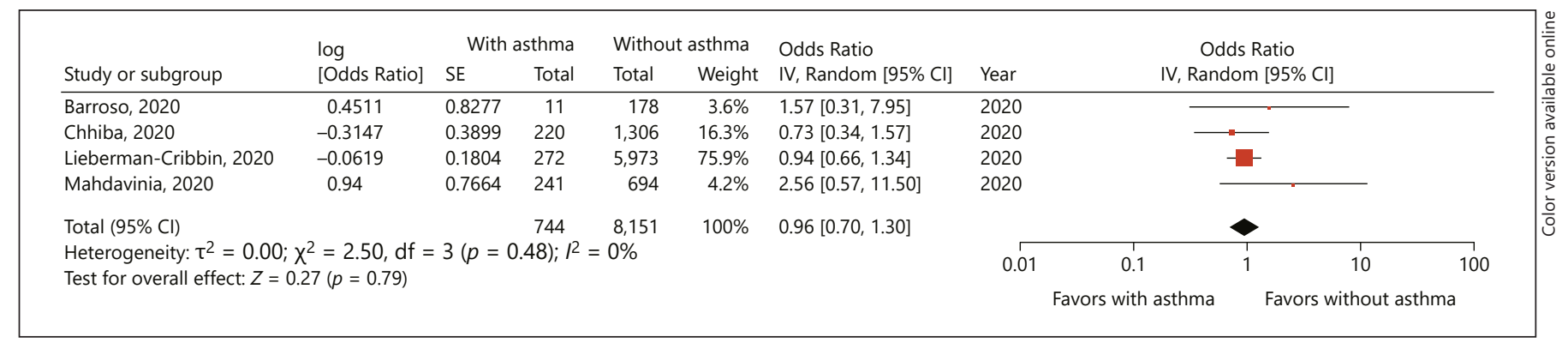

Fig. 2. Forest plot of mortality with COVID-19 with and without asthma $[13-15,17]$. IV, instrumental variable.

Data Extraction and Quality of the Included Studies

A data abstraction form was used to extract the following details: authors, publication year, study type, country of origin, demographic data, comorbidities and smoking, history of corticosteroid use, number of patients hospitalized, requirement of intubation, and study outcomes. The primary outcome of interest was to compare the mortality of COVID-19 patients with asthma versus nonasthmatics. All other outcomes reported by the included studies were analyzed descriptively. For assessing the risk of bias, the risk of a bias assessment tool for nonrandomized studies (RoBANS) was used [11]. Studies were graded for each domain as having a low, high, or unclear risk of bias.

\section{Statistical Analysis}

The software Review Manager (RevMan, version 5.3; Nordic Cochrane Centre [Cochrane Collaboration], Copenhagen, Denmark; 2014) was used for the meta-analysis. Mortality data were summarized using the Mantel-Haenszel OR with 95\% CI in a random-effects model. When the OR was reported in the included study, the data was directly collected; otherwise, the OR was calculated using the meta-analysis software itself by entering the number of deaths and the sample size. Heterogeneity was calculated using the $I^{2}$ statistic. $I^{2}$ values of $25-50 \%$ represented a low heterogeneity, values of $50-75 \%$ represented a medium heterogeneity, and values $>75 \%$ represented substantial heterogeneity. A sensitivity analysis was conducted to assess the influence of each study on the overall estimate by excluding one study at a time. Publication bias was assessed by visual inspection of funnel plots only if $>10$ studies were included in the analysis.

\section{Results}

The PRISMA (Preferred Reporting Items for Systematic Reviews and Meta-analyses) [12] flow chart of this review is presented in Figure 1. A total of 324 unique records were identified. After a full-text analysis, a total of 5 studies met the inclusion criteria [13-17]. The characteristics of the studies included in this review are presented in Table 1 . All 5 were published after peer review and no preprints were included in this study. The author's judgment of the risk of bias in the included studies is presented in online supplementary Table 1 (see www.karger. com/doi/10.1159//000510953 for all online suppl. material). Three studies reported data from the USA $[14,15$, 17], 1 reported data from Spain [13], and the other reported data from France [16]. A total of 767 asthmatic patients were compared with 8,234 nonasthmatic patients in the included studies. None of the studies reported statistically significant differences in the age of the study cohorts. Three reported data on other comorbidities; among those, Chhiba et al. [14] reported a significantly higher number of comorbidities in the asthmatic group. The same study also reported a higher number of patients with obesity and current smokers in the asthmatic cohort. In 2 studies [13, 16], all patients in both groups were hospitalized, while in another $2[14,17]$ both hospitalized and nonhospitalized patients were included in the study groups. Three studies $[13,16,17]$ reported data on the number of intubated patients, with none reporting significant differences between the study groups. The use of corticosteroids in the asthma group varied from 4.34 to $45.45 \%$.

\section{Outcomes}

Mortality data was reported by 4 studies [13-15, 17]. A meta-analysis of data from 744 asthmatic patients and 8,151 nonasthmatic patients indicated that presence of asthma had no significant effect on mortality $\left(\mathrm{OR}=0.96 ; 95 \%\right.$ CI $\left.0.70-1.30 ; I^{2}=0 \% ; p=0.79\right)$ (Fig. 2). The results of the sensitivity analysis are presented in Table 2. There was no change in the significance of the results after the singular exclusion of the included studies.

The details of other outcomes reported by the studies are presented in Table 3 . Three studies $[13,16,17]$ as- 
Table 2. Sensitivity analysis

\begin{tabular}{ll}
\hline Excluded study & Effect size \\
\hline Barroso et al. [13] & OR $=0.94 ; 95 \%$ CI $0.67-1.34 ; I^{2}=6 \% ; p=0.74$ \\
Chhiba et al. [14] & OR $=1.01 ; 95 \%$ CI $0.72-1.42 ; I^{2}=0 \% ; p=0.95$ \\
Lieberman-Cribbin et al. [15] & OR $=1.10 ; 95 \%$ CI $0.52-2.34 ; I^{2}=18 \% ; p=0.79$ \\
Mahdavinia et al. [17] & OR $=0.92 ; 95 \%$ CI $0.67-1.26 ; I^{2}=0 \% ; p=0.59$ \\
\hline
\end{tabular}

Table 3. Other outcomes assessed by the included studies

\begin{tabular}{|c|c|}
\hline Study & Outcomes other than mortality \\
\hline Barroso et al. [13] & $\begin{array}{l}\text { 1. Duration of hospitalization: with asthma, } 9.72 \pm 8.14 \text { days; without asthma, } 10.9 \pm 9.67 \text { days; no statistical } \\
\text { significant difference between the } 2 \text { groups } \\
\text { 2. Transfer to the ICU: with asthma, } 2(18.18 \%) \text {; without asthma, } 30 \text { (16.85\%); no statistical significant } \\
\text { difference between the } 2 \text { groups }\end{array}$ \\
\hline Chhiba et al. [14] & $\begin{array}{l}\text { 1. Risk of hospitalization: } \mathrm{RR}=0.96 ; 95 \% \mathrm{CI} 0.77-1.19 \text {; asthma was not associated with an increased risk } \\
\text { of hospitalization }\end{array}$ \\
\hline Grandbastein et al. [16] & $\begin{array}{l}\text { 1. Prolonged hospital stay: } \mathrm{OR}=1.614 ; 95 \% \mathrm{CI} 0.58-4.58 \text {; asthma was not associated with a prolonged } \\
\text { hospital stay } \\
\text { 2. Transfer to the ICU: OR }=1.06 ; 95 \% \text { CI } 0.27-3.52 \text {; asthma was not associated with a higher risk of } \\
\text { transfer to the ICU }\end{array}$ \\
\hline Mahdavinia et al. [17] & $\begin{array}{l}\text { 1. Duration of hospitalization: with asthma, } 8.36 \pm 6.02 \text { days; without asthma, } 7.81 \pm 5.18 \text { days; no statisti- } \\
\text { cally significant difference was found between the } 2 \text { groups } \\
\text { 2. Duration of intubation: with asthma, } 10.17 \pm 6.91 \text { days; without asthma, } 5.29 \pm 5.98 \text { days; significantly } \\
\text { prolonged duration of intubation in asthmatics } \\
\text { 3. ARDS: with asthma, } 21(8.9 \%) \text {; without asthma, } 65(9.5 \%) \text {; no statistical significant difference between } \\
\text { the } 2 \text { groups }\end{array}$ \\
\hline
\end{tabular}

ARDS, acute respiratory distress syndrome.

sessed the duration of hospitalization between the 2 groups, with none reporting a prolonged hospital stay with asthma. Data on the risk of intensive care unit (ICU) transfer was reported by 2 studies $[13,16]$ and neither of them found any significant difference between asthmatics and nonasthmatics. Mahdavinia et al. [17] studied the risk of acute respiratory distress syndrome in both cohorts and reported no statistically significant difference; however, they found a significantly prolonged duration of intubation in asthmatics. Chhiba et al. [14] reported no difference in the risk of hospitalization between asthmatics and nonasthmatics.

\section{Discussion}

Based on analysis of data from a limited number of studies, our results indicate that asthma may not increase the mortality of patients with COVID-19. A descriptive analysis of further limited evidence suggests that asthma as a comorbidity may not have a major role in prolongation of the hospital stay or an increase in the risk of ICU transfer.

The rising number of COVID-19 cases has overwhelmed the healthcare setups worldwide, and the focus is now gradually shifting to contain the number of deaths due to the disease. Reports from several countries have indicated that the presence of a comorbidity significantly increases the number of deaths in patients with COVID-19 [18-20]. Kim et al. [18], in their study of 2,491 COVID-19 patients, reported that patients with 3 or more underlying conditions had a 1.3 times higher risk of ICU admission and a 1.8 times higher risk of in-hospital mortality. Comorbidities like hypertension, diabetes mellitus, chronic obstructive pulmonary disease, malignancy, obesity, immunosuppression, and renal disease have been found to increase the risk of mortality in patients with COVID-19 $[18,19]$. In this context, the role of asthma in
80

Int Arch Allergy Immunol 2021;182:76-82 DOI: $10.1159 / 000510953$
Wang/Chen/Chen/Liu/Dong/Ji/Hu/ Zhang 
influencing COVID-19-related outcomes is, however, unclear.

Upon systematic examination of the literature, we found only 5 studies carrying out a head-on comparison of outcomes in asthmatics versus nonasthmatics. A pooled analysis of data indicated that the presence of asthma did not significantly increase the odds of death as compared to patients without asthma. The finding of our meta-analysis was stable on sensitivity analysis, with no change in the significance of exclusion of any of the included studies. A detailed analysis of the forest plot indicates that none of the included studies reported any statistically significant difference between the 2 groups. While interpreting the results of the pooled analysis, it is important to examine the presence of other factors influencing mortality. In addition to underlying medical conditions, an older age has been strongly associated with an increased risk of hospitalization, ICU transfer, and death in patients with COVID-19 $[18,21]$. Of the 5 studies in this review, none reported any statistically significant difference in the age of the 2 cohorts; however, in the study of Chhiba et al. [14], asthmatic patients had a significantly higher prevalence of other comorbidities as compared to nonasthmatics. Despite this difference, they reported no difference in mortality between the 2 groups. The results of our analysis are in contrast with recently published data from NHS England [22]. In a report of 5,683 COVID-19-linked deaths, the authors reported a higher probability of death in asthmatics with $(\mathrm{HR}=1.25 ; 95 \%$ CI 1.08-1.44) and without corticosteroid use (HR = 1.11; 95\% CI 1.02-1.20). It is important to note that the lower end of the $95 \%$ CI was just above 1 in their study, indicating a small difference between asthmatics and nonasthmatics [22]. The investigators did not divide the study cohort based on the presence of asthma, and hence the data was not included in this review.

Details of other outcomes like the length of hospital stay, the risk of ICU transfer, the duration of intubation, etc., were not uniformly reported by the included studies. Of the limited data available, statistically significant results were reported only by Mahdavinia et al. [17] for the duration of intubation, which was increased in asthmatics. Data from previous SARS epidemics have produced contrasting results in terms of the influence of asthma on the outcomes of such respiratory infections. Studies on the 2003 SARS epidemic have reported that asthmatics had a reduced susceptibility to the coronavirus with a good overall prognosis [23]. On the other hand, data from the $2009 \mathrm{H} 1 \mathrm{~N} 1$ pandemic indicates that asthma was associated with more severe disease and an increased need for invasive ventilation [24]. Few studies not included in this review have assessed the influence of asthma and disease severity. Zhu et al. [25], in an analysis of 641 COVID19-positive patients, reported an increased disease severity in asthmatics (OR = 1.39; 95\% CI 1.13-1.71). Similarly, Mendy et al. [26], in a report of 689 patients, found higher odds of disease severity (OR $=3.11$; 95\% CI 1.67 5.80) in asthmatics versus nonasthmatics. Baseline data of patients with and without asthma was, however, not available from these studies.

Our review has some limitations. Foremost, data was sourced only from a limited number of studies in this review. Furthermore, the total number of asthmatics in the 2 studies was less than 25 . Secondly, our analysis represents pooled data from retrospective studies, which limits our conclusions to associations rather than causal inferences. Thirdly, asthma was self-reported in all studies. It is possible that asthma may not have been adequately recorded in the medical charts of all of the patients. Also, the included sample did not represent the entire COVID-19 load of the geographical region. A preference for testing for severe cases may have eliminated patients with less severe disease from the analysis. Fourthly, the influence of confounding variables like asthma severity, use of corticosteroids, and severity of COVID-19 was not assessed in our analysis. This may have skewed the overall results. Lastly, not all outcomes were assessed in the included studies. This limited our review to analysis of only mortality data and performance of a descriptive analysis for others.

Nevertheless, our study presents the first meta-analysis analyzing the influence of asthma on outcomes of COVID-19 patients. Preliminary data indicate that asthma as a comorbidity may not increase the mortality of COVID-19. Data on the influence of asthma on the risk of hospitalization, the duration of hospitalization, the requirement of ICU admission, and disease severity is still too limited to draw any strong conclusions. Further studies with a larger sample size are required to establish strong evidence.

\section{Conflict of Interest Statement}

The authors declare no conflict of interests.

\section{Funding Sources}

None. 


\section{Author Contributions}

Y.W. conceived and designed this study. JC, WC, LL, MD, JJ, and $\mathrm{DH}$ collected the data and performed the literature search. N.Z. was involved in the writing of this paper. All of the authors read and approved the final version of this paper.

\section{Statement of Ethics}

This systematic review and meta-analysis followed the guidelines of the PRISMA statement.

\section{References}

1 World Health Organization. Coronavirus situation report. Available from: https://www. who.int/docs/default-source/coronaviruse/ situation-reports/20200407-sitrep-78-covid-19.pdf?sfvrsn=bc43e1b_2.

2 Wang D, Hu B, Hu C, Zhu F, Liu X, Zhang J, et al. Clinical Characteristics of 138 Hospitalized Patients With 2019 Novel CoronavirusInfected Pneumonia in Wuhan, China. JAMA. 2020 Feb;323(11):1061.

3 Jiang Y, He S, Zhang C, Wang X, Chen X, Jin $\mathrm{Y}$, et al. Clinical characteristics of 60 discharged cases of 2019 novel coronavirus-infected pneumonia in Taizhou, China. Ann Transl Med. 2020 Apr;8(8):547-547.

4 Arentz M, Yim E, Klaff L, Lokhandwala S, Riedo FX, Chong M, et al. Characteristics and Outcomes of 21 Critically Ill Patients With COVID-19 in Washington State. JAMA. 2020 Mar;323(16):1612-4.

5 Richardson S, Hirsch JS, Narasimhan M, Crawford JM, McGinn T, Davidson KW, et al.; and the Northwell COVID-19 Research Consortium. Presenting Characteristics, Comorbidities, and Outcomes Among $5700 \mathrm{~Pa}$ tients Hospitalized With COVID-19 in the New York City Area. JAMA. 2020 Apr; 323(20):2052-9.

6 Zhou Y, Liu Y. Recent trends in current asthma prevalence among US adults, 2009-2018. J Allergy Clin Immunol Pract. 2020 Apr; S2213-2198(20)30398-6

7 Papadopoulos NG, Christodoulou I, Rohde G, Agache I, Almqvist C, Bruno A, et al. Viruses and bacteria in acute asthma exacerbations-a GA ${ }^{2}$ LEN-DARE systematic review. Allergy. 2011 Apr;66(4):458-68.

8 Morais-Almeida M, Pité H, Aguiar R, Ansotegui I, Bousquet J, Aguiar R, et al. Asthma and the coronavirus disease 2019 pandemic: a literature review. Int Arch Allergy Immunol. 2020;181:680-8.

9 Zhang JJ, Dong X, Cao YY, Yuan YD, Yang YB, Yan YQ, et al. Clinical characteristics of 140 patients infected with SARS-CoV-2 in Wuhan, China. Allergy. 2020 Jul;75(7):173041.

10 Centers for Disease Control and Prevention. People with moderate to severe asthma. [cited 2020 Jul 1]. Available from: https://www.cdc. gov/coronavirus/2019-ncov/need-extra-precautions/asthma.html.
11 Kim SY, Park JE, Lee YJ, Seo HJ, Sheen SS, Hahn S, et al. Testing a tool for assessing the risk of bias for nonrandomized studies showed moderate reliability and promising validity. J Clin Epidemiol. 2013 Apr;66(4): 408-14.

12 Moher D, Liberati A, Tetzlaff J, Altman DG; PRISMA Group. Preferred reporting items for systematic reviews and meta-analyses: the PRISMA statement. PLoS Med. 2009 Jul; 6(7):e1000097.

13 Barroso B, Valverde-Monge M, Cañas JA, Rodrigo-Muñoz JM, Gonzalez-Cano B, Villalobos-Violan V, et al.; COVID FJD-TEAM. Presenting prevalence, characteristics and outcome of asthmatic patients with T2 diseases in hospitalized subjects with COVID-19 in Madrid, Spain. J Investig Allergol Clin Immunol. 2020 Jun;30(5):0.

14 Chhiba KD, Patel GB, Vu TH, Chen MM, Guo A, Kudlaty E, et al. Prevalence and characterization of asthma in hospitalized and nonhospitalized patients with COVID-19. J Allergy Clin Immunol. 2020 Aug;146(2):307314.e4.

15 Lieberman-Cribbin W, Rapp J, Alpert N, Tuminello S, Taioli E. The impact of asthma on mortality in patients with COVID-19. Chest. 2020. doi: 10.1016/j.chest.2020.05.575.

16 Grandbastien M, Piotin A, Godet J, AbessoloAmougou I, Ederlé C, Enache I, et al. SARSCoV-2 pneumonia in hospitalized asthmatic patients did not induce severe exacerbation. J Allergy Clin Immunol Pract. 2020. doi: 10.1016/j.jaip.2020.06.032.

17 Mahdavinia M, Foster KJ, Jauregui E, Moore D, Adnan D, Andy-Nweye AB, et al. Asthma prolongs intubation in COVID-19. J Allergy Clin Immunol Pract. 2020 Jul - Aug;8(7): 2388-91.

18 Kim L, Garg S, Halloran A, Whitaker M, Pham $\mathrm{H}$, Anderson EJ, et al. Interim analysis of risk factors for severe outcomes among a cohort of hospitalized adults identified through the US Coronavirus Disease 2019 (COVID-19)-Associated Hospitalization Surveillance Network (COVID-NET). medRxiv. doi: 10.1093/cid/ciaa1012.
19 Guan WJ, Liang WH, Zhao Y, Liang HR, Chen ZS, Li YM, et al.; China Medical Treatment Expert Group for COVID-19. Comorbidity and its impact on 1590 patients with COVID-19 in China: a nationwide analysis. Eur Respir J. 2020 May;55(5):2000547.

20 Petrilli CM, Jones SA, Yang J, Rajagopalan H, O'Donnell L, Chernyak Y, et al. Factors associated with hospital admission and critical illness among 5,279 people with coronavirus disease 2019 in New York City: prospective cohort study. BMJ. 2020 May;369:m1966.

21 Chen T, Wu D, Chen H, Yan W, Yang D, Chen $\mathrm{G}$, et al. Clinical characteristics of 113 deceased patients with coronavirus disease 2019: retrospective study. BMJ. 2020 Mar; 368:m1091.

22 Williamson EJ, Walker AJ, Bhaskaran K, Bacon S, Bates C, Morton CE, et al. OpenSAFELY: factors associated with COVID-19 death in 17 million patients. Nature. 2020. doi: 10.1038/s41586-020-2521-4.

23 Halpin DM, Faner R, Sibila O, Badia JR, Agusti A. Do chronic respiratory diseases or their treatment affect the risk of SARS-CoV-2 infection? Lancet Respir Med. 2020 May;8(5): 436-8.

24 Tokuhira N, Shime N, Inoue M, Kawasaki T, Sakurai Y, Kurosaka N, et al.; Writing Committee of AH1N1 Investigators; Japanese Society of Intensive Care Medicine Pediatric Intensive Care Unit Network. Mechanically ventilated children with 2009 pandemic influenza A/H1N1: results from the National Pediatric Intensive Care Registry in Japan. Pediatr Crit Care Med. 2012 Sep;13(5):e2948.

25 Zhu Z, Hasegawa K, Ma B, Fujiogi M, Camargo CA Jr, Liang L. Association of asthma and its genetic predisposition with the risk of severe COVID-19. J Allergy Clin Immunol. 2020 Aug;146(2):327-329.e4.

26 Mendy A, Apewokin S, Wells AA, Morrow AL. Factors associated with hospitalization and disease severity in a racially and ethnically diverse population of COVID-19 patients. medRxiv. 2020. doi: 10.1101/2020. 06.25.20137323. 